\title{
AN ARTIFICIAL INTELLIGENCE APPLICATION IN PORTFOLIO MANAGEMENT
}

\author{
Xiaoqiang Zhang \\ School of Management and Science, Nanjing University, P.R. China \\ Ying Chen* \\ School of Management and Science, Nanjing University, P.R. China
}

\begin{abstract}
Artificial intelligence (AI) aims to supplement and even substitute for practically all tasks currently performed by humans. Under the drive of external forces and technological advances, AI is used in various domains. Among these, AI applications in finance is facing a promising future. This paper reviews AI applications in different areas, especially in financial industry and points out the core techniques and specific scenes. This paper also take the AI application in portfolio management as an example, construct a portfolio based on spectral clustering (SC) connected to stock complex network. The portfolio constructed by AI techniques in this example proves to outperformance its traditional counterparts.
\end{abstract}

Keywords: artificial intelligence, spectral clustering, application, finance JEL: C8, G13

\section{Introduction}

As an important branch of computer science, artificial intelligence has gained more and more attention in the modern society. We have witnessed the emerging of many artificial intelligent technologies such as computer vision, natural language processing, robotics. Artificial Intelligence is very much anticipated to contribute in solving problems under multi-dimensional system including ecological protection, economic management, urban development and financial risk. Wide range of specific issues from product design to safe driving, medical treatment to energy conservation are all provided with advanced artificial intelligence solutions.

In fact, over the past decades, numerous predictions have been made about artificial intelligence revolution and its impact on every aspects in both individual and business 
entity life. But it is not until recent years that artificial intelligence has gained rapid development. Pan Y(2016), Makridakis S(2017) conclude two main engines that drive the development of AI: (a) external forces such as information environment and social needs changing the goal of artificial intelligence appliance; (b) emergence of new AI techniques: Pan Y(2016) summarizes five important technological advances: big-databased AI, internet crowd intelligence, cross-media intelligence, human-machine hybrid-augmented intelligence and autonomous-intelligent systems. Problems generated in various domains caused growing interest in artificial intelligent techniques and are also requiring higher advance in this emerging discipline.

S.M. Zahraee(2016) analyzes how to maximize the use of renewable energy resources. He presents a thorough analysis of artificial intelligence application that penetrate extensively into every aspects of renewable energy, and discuss how AI helps to economically improve renewable systems. Hamet \& Tremblay (2017) review the history of AI applications in medicine, and categorized current researches into two main branches: virtual and physical. Virtual branch is mainly informaticas approaches aiming to better operate the health management systems, while physical branch aims to assist the patients and surgeons with physical intelligent robots. AI help promote the development of both branches. Qian Sun, Turgay Ertekin(2016) study the artificial intelligence in order to optimize a specific engineering technology-CSS(Cyclic steam stimulation), and concluded that artificial neural network (ANN) based model is a powerful subsidiary tool for its fast computational speed and reliable prediction capability. Qian also use a network topology optimization workflow to help ANN better understand the complex data structures that are encountered in CSS processes, and prove a nice outperform. Jui-sheng Chou(2016) apply different artificial intelligence techniques in predicting corrosion rate, including artificial neural networks (ANNs), support vector regression/machines (SVR/SVMs), classification and regression tree (CART), and linear regression (LR), they conclude that Hybrid Metaheuristic Regression Model which combine a smart nature-inspired metaheuristic optimization algorithm and a least squares SVR is a promising and practical methodology. M. S.(2016) analyze the robust, reliable, and fast performance of artificial intelligencebased MPPT methods, and utilized the method for the integration of photovoltaic (PV) systems to grid-connected or isolated micro-grids. S.W. Tsang(2016) employs artificial intelligence algorithms composed of artificial neural network and fuzzy logic to build an optimal irrigation strategy. Weather data is modeled through algorithms to simulate soil moisture changes. Mariusz Mlynarczuk(2017) use pattern recognition techniques $(\mathrm{NN}, \mathrm{kNN})$ to identify the maceral groups of coal, i.e. vitrinite, inertinite, liptinite, along with some other non-organic minerals. 
Except for the applications in industrial field, artificial intelligence is gaining more popularity especially in finance. A well functioned finance sector is always tightly correlated with the well-being of a society and the development of individuals enrolled. Promoting and optimizing capital accumulation is one of the most crucial sessions that researches focus on. Different methods are introduced to predict economic trend, manage asset portfolio, stimulate investor's behavior, optimize capital structure, create credit products, and so forth Arash Bahrammirzace(2010) categorized available financial market researching methods into three categories: parametric statistical methods (e.g. discriminant analysis), nonparametric statistical methods (e.g. k nearest neighbor) and soft-computing approaches (e.g. artificial intelligent algorithms). Among these methods, artificial intelligent algorithms have been given more attention, methods such as ANNs (Artificial neural network), ANFIS (adaptive network based fuzzy inference system), decision trees etc. have been used in many financial situations including financial prediction and planning, credit evaluation, portfolio management, etc. These methods prove to have either better forecasting accuracy or have higher operation efficiency.

This paper is focused on finance application in artificial intelligence. We summarize artificial intelligence methods available and their corresponding appliance area. One specific case on applying artificial intelligence on financial industry is given.

The remainder of the paper is organized as follows.: section 2 presents a brief overview of the artificial intelligence applications in finance. An example of how to adopt artificial intelligence in portfolio management is provided in section 3. Here we use spectral clustering based on complex network to select the stock portfolio and measure its performance. Finally, section 4 concludes.

\section{Artificial intelligence applications in finance}

Generally saying, artificial intelligence has a broad prospect in financial industry. We conclude them into three parts as following: portfolio management, credit assessment, Financial forecasting,

\subsection{Portfolio Management}

Techniques, mainly algorithms, to determining optimal diversified asset allocation is a mainstream research area. Among these algorithms the most famous is ANNs, since the investor's decision process is unstructured and the market environment is uncertain, the financial market is a somewhat complex nonlinear dynamic system. ANNs can seize the nonlinear characteristic properly, and is surely a suitable method to do portfolio 
management. ANNs has been successfully applied in different markets, such as Mortgage market, stock market, real estate market, accounting, and is proved to have higher performance than traditional models.

Apart from specific algorithms, investors also rely on expert system (ES) based on AI to do investment decision. For example, a fund manager can assign some of the analysis tasks to the ES and the latter will design a knowledge-based portfolio analysis for him. Individual investor can even fully rely on the ES to acquire information and do investment decisions. NPD, INVEX, PORSEL are platforms that provide such service. A knowledge center for information search is included and different methods are provided to make a rating for stocks. Finally, optimal portfolio is generated for investors.

ES is a somewhat static platform, while the decision-making situations that we encounter are always complex and dynamic, especially under numerous conflicting criteria. Therefore, some hybrid portfolio selection models are proposed accordingly. Hybrid methods used commonly are Neuro-fuzzy system, Adaptive ANFIS, Gaussian RBF, neural-based Q-learning, etc. These hybrid methods perform better in most cases, although not overall superior to other alternative ones, and result in higher earnings for investment. Introducing leaning ability into algorithms is another branch of improved approach. For example, the Robo-Advisor, which is a complex artificial intelligent system with the ability of self-learning and self-enhancing. Take the most famous "Robo-Advisor" company Wealthfront for example, it manages up to 2 billion dollars by 2016 and outperform than many other traditional investment management companies.

\subsection{Credit Assessment}

Credit is an invisible property for personal or corporate entity. Credit scoring is a technique invented and used for the process of quantification on credit. Credit assessment through credit scoring are usually seen as a linear process and a categorization problem in most of the cases. However, one's credit constitutes far more than one dimension of evaluating. Requiring higher accuracy and lower risk, the credit assessment industry is paying more attention on artificial intelligence algorithms. The most commonly used method must be ANNs. Other algorithms like BPNN, DRPNN, CFLANN are then developed on it. Also there are other methods such as decision trees and support vector machine (SVM). Artificial intelligence methods are proved to be superior than most of the traditional methods, especially in regards of accuracy. 
As introduced before, expert systems based on these methods are built to seek for accuracy and speed, and they are used in numerous scenes, like FINISM for industrial companies credit assessment, ALEES for agricultural credit evaluation, VPRS for financial institutions credit assessment. Furthermore, in the researches available, all these expert systems are proved to have better effectiveness than traditional methods. Hybrid systems with those methods are also paied high concerns, and are excepted to have outstanding effect in less usual situations.

\subsection{Financial Prediction}

Financial forecasting is also an promising area in artificial intelligence application. Considering the inherent nonlinearities of financial data, the problem of how to extract useful information from a market filled with unstructured data and how to accurately forecast asset price is becoming an extremely challenging task. AI techniques such as ANN, ANFIS, decision trees can solve these problems efficiently. They are able to detect the dynamic characteristic of the data and adopt them into the predicting model, their advance in selecting the optimal training window further devote to a better result.

Binoy B. Nair(2015) concludes several financial predicting domains that require artificial intelligence: forecasting exchange rates, decision support systems, analysis of the behavior of various financial time series, stock index and stock price forecasting.

Techniques in this discipline are various, we concludes the available methods as following: (a) ANNs: such as ENNs used for stock price prediction. RBFNNs used for exchange rate prediction, TDNNs used for index prediction. (b) support vector machines(SVMs), SVM is used to separate data into two classes. this method can seize the nonlinear characteristic and predict the index or price movement. (c) Fuzzy logic and adaptive network based fuzzy inference system (ANFIS): though build up an efficient fuzzy system is challenging, this method is more robust than other methods, and is suitable for price prediction. (d) Evolutionary algorithms (EAs), such as GP(genetic program) used for exchange rate prediction, PSO (particle Swarm Optimization) used for feature selection.

\subsection{Discussion}

As it is illustrated above, artificial intelligence is seemingly theoretically profound. However, AI applications in finance still require specific condition. Artificial intelligence as a tool plays a complementary role rather than substitution role. Therefore, if we are to further develop artificial intelligence technique in financial industry, there are two critical breakpoints: one is to discover more scenarios to adapt AI techniques, 
meanwhile further optimize existing application; the other is to try more new artificial intelligence algorithms and optimize the result, embracing more breakthroughs.

The example we give below is a specific application in algorithm study in portfolio management. With the prevailing trend of personal wealth management, especially passive investing strategy (buying ETFs for example), portfolio management is a mainstream research area in financial study. The finding calls for more innovational appliance of AI in finance, which is theoretically and economically meaningful.

\section{An example: portfolio management based on Spectral Clustering (SC)}

In this section, we give an example of how artificial intelligence can be used to optimize portfolio management. We choose SC based on stock complex network to construct a portfolio, and measure the performance of the portfolio.

\subsection{Traditional portfolio management Based on Stock Network}

Stock market is a typical complex system, in which the cross-correlations among financial units are particularly important for understanding this interactive mechanism and thus optimizing portfolio selections. In the past few years, network models are applied in researches to reveal the information beneath internal correlations among stocks, such as the minimum spanning tree (MST), the planar maximal filtering graph(PMFG), and the correlation threshold methods. Models are adopted to analysis the market as a whole and, meanwhile, construct portfolios according to factors generated from the stock network. Pozzi(2013) studies stock centrality and prove that low centrality stocks, which means the marginalized stocks in the stock network, bring higher absolute return. We do the same test here and prove this. We set the data set on HS300 stocks (Jan $4^{\text {th }} 2013$ - Dec $31^{\text {st }} 2016$ ), and add the centrality factor into FamaFrench three-factor model to make the four-factor model as below:

$$
R_{i t}-R_{f t}=\alpha_{i t}+\beta_{i t}\left(R_{m t}-R_{f t}\right)+s_{i t} S M B_{t}+h_{i t} H M L_{t}+k_{i t} D M G_{t}+\varepsilon_{i t}
$$

where: $1 . R_{f t}$ refers to a riskless investment return, which would be denoted as the interest rate of the one-year deposit;

2. $R_{m t}$ denotes market return at time $\mathrm{t}$, which would be calculated by HS300 index;

3. $R_{i t}$ denotes asset return at time t, where $R_{m t}-R_{f t}$ refers to market risk premium and $R_{i t}-R_{f t}$ the portfolio premium;

4. $S M B_{t}$ (Small minus Big) refers to the historic excess returns of small caps over big caps, calculated as the difference between the returns on small-stock and big-stock 
portfolios composed by ranked stocks on book-to-market equity;

5. $H M L_{t}$ (High minus low) refers to the historic excess returns of value stocks over growth stocks, calculated as the difference between the returns on high and low bookto-market equity portfolios composed by ranked stocks on average size;

6. $D M G_{t}$ refers to the historic excess returns of value stocks over growth stocks, calculated as the difference between the returns on high and low centrality portfolios composed by ranked stocks on centrality in the network;

7. $\alpha$ denotes a constant of excessive return that cannot be explained by the factors above.

The result is as following:

Table 1 Four-factors regression results

\begin{tabular}{|c|c|c|c|c|c|c|c|c|}
\hline & Portfol & & $\alpha$ & $r_{m}-r_{f}$ & SMB & HML & DMG & $R^{2}$ \\
\hline \multirow[t]{4}{*}{$\begin{array}{c}\text { High } \\
\text { degree }\end{array}$} & \multirow[t]{2}{*}{$\begin{array}{c}\text { High } \\
\text { size }\end{array}$} & High BM & $\begin{array}{l}2.081^{* * *} \\
(5.800)\end{array}$ & $\begin{array}{l}1.087^{* * *} \\
(23.170)\end{array}$ & $\begin{array}{l}-0.455^{* * *} \\
(-3.435)\end{array}$ & $\begin{array}{l}0.547^{* * *} \\
(9.475)\end{array}$ & $\begin{array}{l}-0.344^{* * *} \\
(-5.610)\end{array}$ & 0.969 \\
\hline & & Low BM & $\begin{array}{l}-0.735 \\
(-0.582)\end{array}$ & $\begin{array}{l}1.113^{* * *} \\
(5.825)\end{array}$ & $\begin{array}{l}-0.241 \\
(-0.675)\end{array}$ & $\begin{array}{l}-0.276 \\
(-1.534)\end{array}$ & $\begin{array}{c}0.355 \\
(1.586)\end{array}$ & 0.724 \\
\hline & \multirow[t]{2}{*}{$\begin{array}{l}\text { Low } \\
\text { size }\end{array}$} & High BM & $\begin{array}{l}1.288^{* * *} \\
(3.331)\end{array}$ & $\begin{array}{l}0.976^{* * *} \\
(19.303)\end{array}$ & $\begin{array}{c}0.585^{* * *} \\
(4.097)\end{array}$ & $\begin{array}{l}0.446^{* * *} \\
(7.159)\end{array}$ & $\begin{array}{l}-0.244^{* * *} \\
(-3.700)\end{array}$ & 0.960 \\
\hline & & Low BM & $\begin{array}{c}0.898 \\
(0.840)\end{array}$ & $\begin{array}{l}1.287^{* * *} \\
(9.210)\end{array}$ & $\begin{array}{l}1.182^{* * *} \\
(2.994)\end{array}$ & $\begin{array}{c}0.017 \\
(0.098)\end{array}$ & $\begin{array}{l}-1.105^{* * *} \\
(-6.053)\end{array}$ & 0.898 \\
\hline \multirow[t]{4}{*}{$\begin{array}{c}\text { Low } \\
\text { degree }\end{array}$} & \multirow[t]{2}{*}{$\begin{array}{l}\text { High } \\
\text { size }\end{array}$} & High BM & $\begin{array}{c}1.080 \\
(1.537)\end{array}$ & $\begin{array}{l}1.120^{* * *} \\
(12.186)\end{array}$ & $\begin{array}{l}-0.408 \\
(-1.57)\end{array}$ & $\begin{array}{l}0.381^{* * *} \\
(3.370)\end{array}$ & $\begin{array}{c}0.428^{* * *} \\
(3.570)\end{array}$ & 0.815 \\
\hline & & Low BM & $\begin{array}{c}0.674 \\
(1.509)\end{array}$ & $\begin{array}{l}1.017^{* * *} \\
(17.438)\end{array}$ & $\begin{array}{l}-0.095 \\
(-0.577)\end{array}$ & $\begin{array}{l}-0.267^{* * *} \\
(-3.710)\end{array}$ & $\begin{array}{l}0.648^{* * *} \\
(8.512)\end{array}$ & 0.902 \\
\hline & \multirow[t]{2}{*}{$\begin{array}{l}\text { Low } \\
\text { size }\end{array}$} & High BM & $\begin{array}{c}0.578 \\
(0.576)\end{array}$ & $\begin{array}{l}1.167^{* * *} \\
(10.008)\end{array}$ & $\begin{array}{l}0.878^{* * *} \\
(2.620)\end{array}$ & $\begin{array}{l}1.262^{* * *} \\
(8.727)\end{array}$ & $\begin{array}{l}0.872^{* * *} \\
(5.833)\end{array}$ & 0.867 \\
\hline & & Low BM & $\begin{array}{l}1.613^{* * *} \\
(3.934)\end{array}$ & $\begin{array}{l}1.107^{* * *} \\
(20.658)\end{array}$ & $\begin{array}{l}0.330^{* *} \\
(2.180)\end{array}$ & $\begin{array}{l}-0.615^{* * *} \\
(-9.311)\end{array}$ & $\begin{array}{l}0.752^{* * *} \\
(10.741)\end{array}$ & 0.944 \\
\hline
\end{tabular}


We can see that centrality is negatively correlated with stock return significantly. Therefore, it is easy to assume that portfolios with low centrality stocks are predicted to perform well. However, those marginalized stocks are mostly small size stocks. Large drawbacks will happen when the market is going down.

We consider all the stocks from Shanghai Stock Exchange and Shenzhen Stock Exchange, dating from January 12009 to April 13 2017, Stock market is very much fluctuated during this sample period. In order to capture the variation, we build dynamical stock networks by moving window method. To be specific, a stock network at a given time is constructed based on the correlation matrix of $T$ trading days before the chosen time point. And the network evolves as the window slides in forward at a $\Delta T$-days interval. A critical question here is the choice of the window length $T$. An unnecessarily large $T$ means the window contains excessive historical data, resulting in a decreasing capacity of the stock network to reflect features of the recent stock market. Conversely, an undersized $T$ means an insufficient sample size of price return sequence, making the correlation coefficient to be unreliable. Given these concerns, we set $T=100$ days in this paper.

$\Delta T$ is the interval between two sampling windows, which is of no significant effect on the network construction. Since there are about 20 trading days in a calendar month, here we set $\Delta T=20$ days. In our previous work, we find the selection of threshold has no significant effect on the fluctuations of network parameters, which possesses certain robustness. During each $T$ trading window, we remove the stocks that is in their trading suspension through the window, that is, suspended from the market for more than T days.

Then we let $\mathrm{P}_{i j}$ be the closing price of stock $\mathrm{i}$ on the day $\mathrm{t}$, and denote the return of the stock price at a time interval $\Delta \mathrm{t}$, which is defined as

$$
r_{i}(t)=\ln p_{i}(t)-\ln p_{i}(t-\Delta t)
$$

We take $\Delta t=1$ day in the following analysis.

Suppose $r_{i}$ and $r_{j}$ are the price returns of stock $\mathrm{i}$ and stock $\mathrm{j}$ respectively. The cross correlation $c_{i j}$ between the two stocks is given by

$$
c_{i j}=\frac{E\left(r_{i} r_{j}\right)-E\left(r_{i}\right) E\left(r_{j}\right)}{\sqrt{\left(E\left(r_{i}^{2}\right)-E^{2}\left(r_{i}\right)\right)\left(E\left(r_{j}^{2}\right)-E^{2}\left(r_{j}\right)\right)}}
$$


For the constructed network, each node represents a stock, and whether there is a link between two nodes depends on a threshold value. Denote graph $G=(V ; E)$ as the network, $\mathrm{V}$ is the set of nodes, and $\mathrm{E}$ is the set of edges. For the purpose of containing more information, we set threshold when building the network, we exclude edges with correlation under $0.7\left(c_{i j}<0.7\right)$ to make the stock network during time T. Target portfolio is generated by bottom 10 stocks of in degree ranking. The following graph is its cumulative return from Jun $5^{\text {th }} 2009$ to Mar $28^{\text {th }}$ 2017. As predicted before, marginalized stocks do perform well before June 2015 however draw back greatly during market crash period.

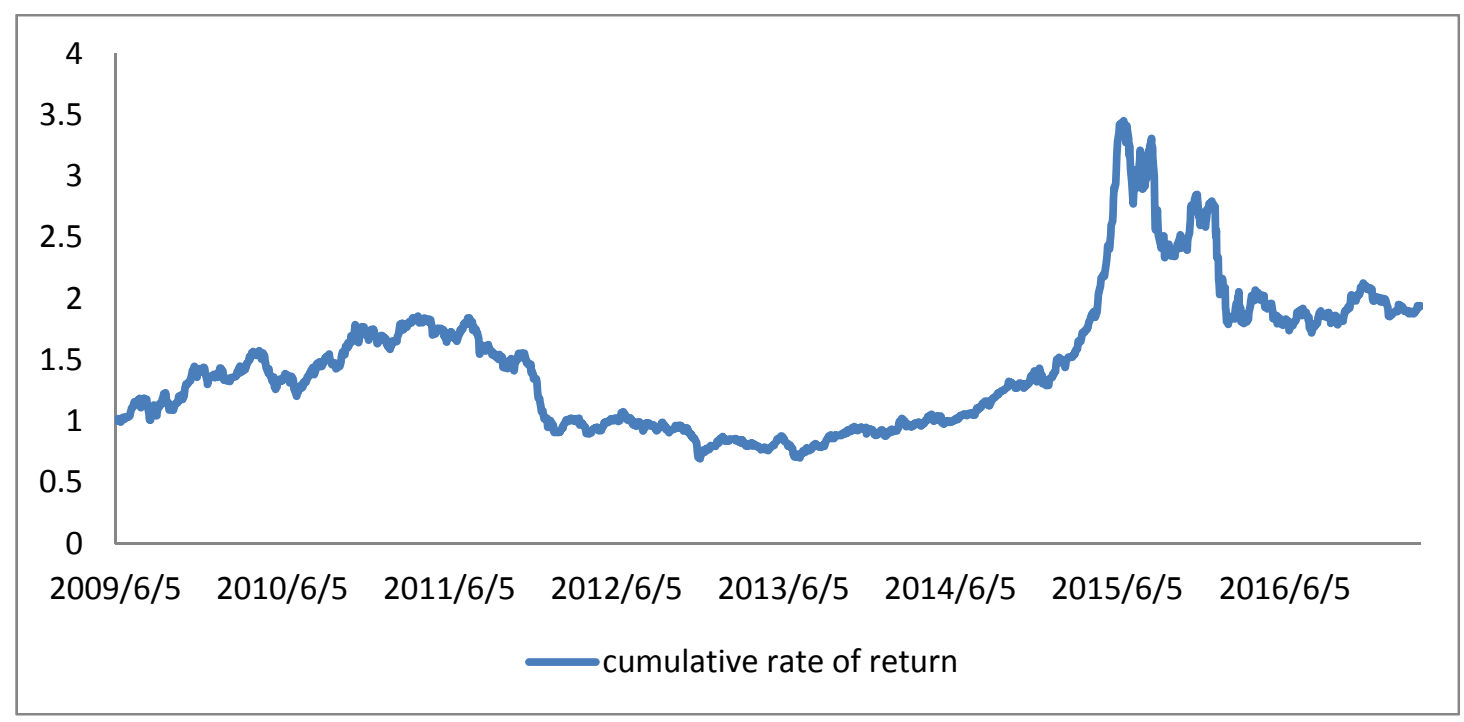

Figure 1 Cumulative return of low centrality portfolio

As illustrated before, traditional network-based portfolio management method is inspired by the fact that marginalized stocks is less correlated with the center stocks and less affected by variation of other stocks, which is proved by our four-factor model here. However, if using threshold method when constructing the stock network, threshold is hard to set; if too much marginalized stocks are included in the portfolio, drawback risk cannot be ignored especially under bear market. With development of artificial intelligence algorithm, we can use this technology to analysis the stock market, and actively response to the diversity and robustness need. We will introduce the details in the next part. 


\section{2 portfolio management based on Spectral Clustering}

$\mathrm{SC}$ techniques is to perform dimensionality reduction before clustering through the spectrum (eigenvalues) of the similarity matrix. The similarity matrix consists of a quantitative assessment of the relative similarity of each pair of points in the dataset and provided as an input.

In this paper, we model Chinese stock market as an evolving complex network, based on daily fluctuations of closing prices for all stocks in Chinese A-share market during 2010 trading day period from January 12009 to April 13 2017. Then we use SC to classify the stocks and construct the portfolio. The model and outcome are given below.

(1) Construction of Stock Network

Similar to part 3.1, we here use a T-day stock network to construct portfolio. The only difference is that we here adopt the original network, which includes every nodes and edges calculated through correlation matrix with no node or edge deleted.

(2) Spectral Clustering of the Network and Building MST

For each $\mathrm{T}$ trading days window, there is a similarity matrix defined as $\mathrm{A}$, where $\mathrm{A}_{i j} \geq$ 0 represents a measure of the similarity between data points with indices $i$ and $j$. The general approach to spectral clustering is to use a standard clustering method on relevant eigenvectors of a Laplacian matrix A.

Since the portfolios we want to build contain 20 stocks, we use SC to divide all the nodes into 20 categories. For each category, we build the MST among them, then we choose the node which has the largest degree in each category.

The reason why we use spectral clustering to construct portfolio is to, on one hand, efficiently diverse and make least correlations between each pair of stocks in the portfolio, and on the other hand, center stock in each cluster is included instead of marginalized stocks. This can avoid large drawback when the market is going down, as well as count in good diversity and robustness.

(3) Performance of the Portfolio

Here we also introduce the Fama-French three-factor model to prove that larger-degree portfolios based on SC generate higher excessive returns as below:

$$
R_{i t}-R_{f t}=\alpha_{i t}+\beta_{i t}\left(R_{m t}-R_{f t}\right)+s_{i t} S M B_{t}+h_{i t} H M L_{t}+\varepsilon_{i t}
$$


And we also use the Reward-to-Variability-Ratio (Sharpe Ratio) to measure the price of risk. This ratio $\mathrm{S}$ of an investment is defined as

$$
\mathrm{S}=\frac{\bar{D}}{\sigma_{D}}
$$

Where $\bar{D}$ is the average excess return against a riskless investment over a time period and $\sigma_{D}$ is the standard deviation of these excess returns. We measure the performance of portfolios composed of the $\mathrm{n}=20$ largest degree stocks in the stock network based on $\mathrm{SC}$ and compared it with other portfolios. Every portfolio is compared with the performance of the market index. Table 2 shows Fama-French constant $\alpha$, Sharpe Ratio and price returns for observed portfolios and HS300 Index. We build two portfolios in total. The first one is the set of stocks with small degrees in dynamic complex network, which is the traditional method. The second one is the portfolio constructed by spectral clustering: nodes are divided into 20 categories, and for each category, we build the MST among them, then node which has the largest degree in each category are collected in this portflio. We can observe that portfolios based on SC systematically outperform other ones. Notably, the performance of portfolios based on SC is comparable and often better than the market performance.

Table2 Performance of different portfolios

\begin{tabular}{|c|c|c|c|}
\hline Portfolio & $\alpha$ & Sharpe Ratio & Return \\
\hline HS 300 index & 0 & 0.219 & $92.8 \%$ \\
\hline portfolio based on SC & 2.452 & 0.235 & $293.2 \%$ \\
\hline Traditional method & 1.692 & 0.188 & $193.4 \%$ \\
\hline
\end{tabular}

We investigate the composition of stocks based on SC, the three indicator considered here are $\mathrm{PE}$ ratio (price-to-earning ratio), $\mathrm{PB}$ ratio (price-to-book ratio) and circulating market value. A high PE ratio implies potential for growing, but the stock is usually over-valued if PE ratio is excessively high. Analysts often deem stocks with low PB ratio to be undervalued, but those with extremely low ratios may just be bad assets. Specifically, we divide stocks from Shanghai and Shenzhen markets into 5 equal groups, according to PE, PB and Circulating Market Cap respectively. As is illustrated in Table 3, large-degree portfolio based on SC tend to select valuable stocks with high growth rate and large book value, and individuals in this portfolio mostly own large circulating market cap, which conflicts with the opinion that the excess return comes from Smallcap Effect in Chinese stock market. Therefore, constructing portfolio based on SC could 
filter out the relatively poor-performing stocks.

Table 3 Probability of PE, PB and circulating market value for large-degree stocks based on SC

\begin{tabular}{|l|c|c|c|c|c|}
\hline & $0-20 \%$ & $20 \%-40 \%$ & $40 \%-60 \%$ & $60 \%-80 \%$ & $80 \%-100 \%$ \\
\hline PE & $9.06 \%$ & $21.22 \%$ & $27.91 \%$ & $25.87 \%$ & $15.94 \%$ \\
\hline PB & $29.20 \%$ & $26.77 \%$ & $20.08 \%$ & $17.56 \%$ & $6.39 \%$ \\
\hline CMV & $8.55 \%$ & $17.16 \%$ & $23.19 \%$ & $26.82 \%$ & $24.28 \%$ \\
\hline
\end{tabular}

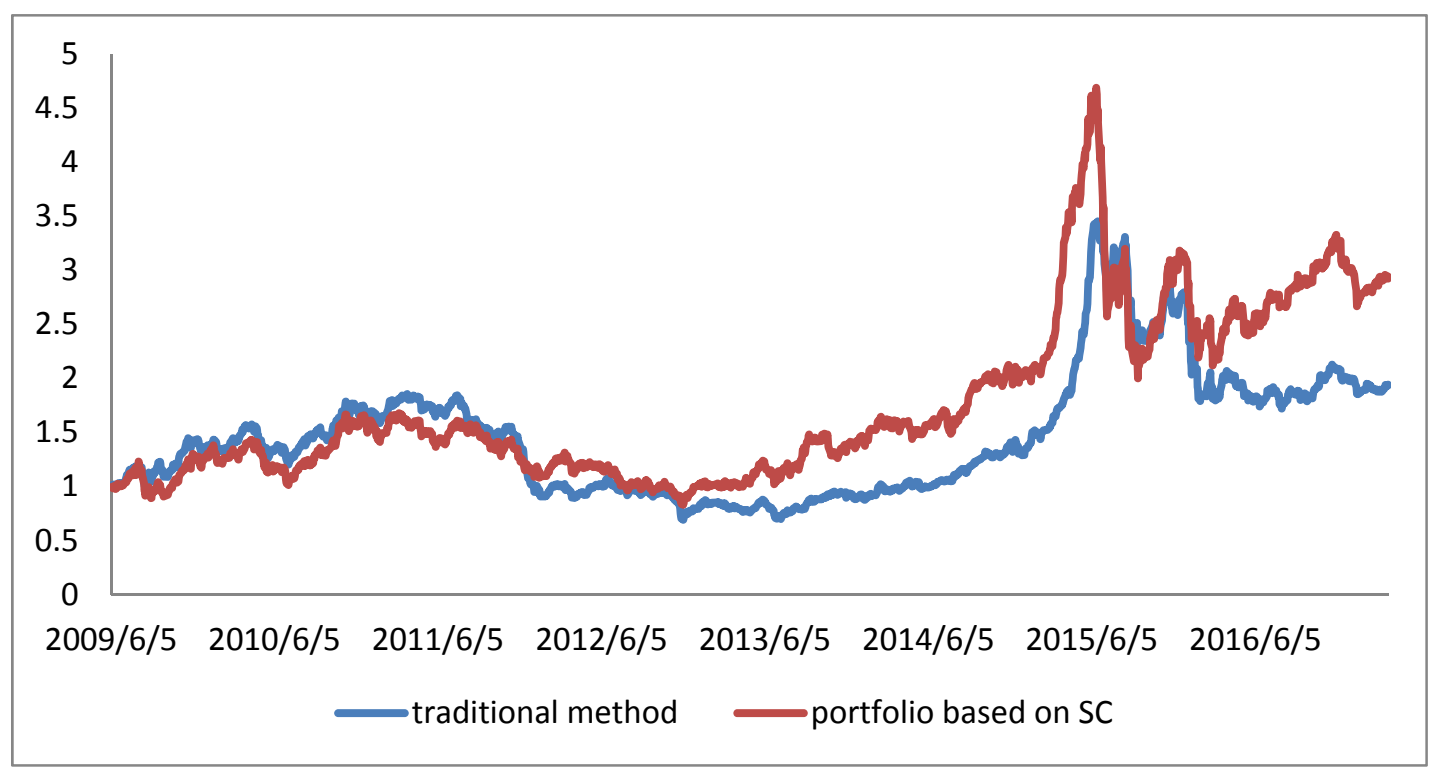

Figure 2. Performance of different portfolios

The graph above is cumulative return of portfolios applying moving window method from 5 June 2009 to 28 March 2017. We can see that portfolios based on SC can get a surprising return of $292.3 \%$ in the whole sample window.

\subsection{Robustness Test}

We alter some of the variables in constructing portfolio to test the robustness of spectral clustering here. We fix the data source window of constructing the stock network at 100 $(\mathrm{T}=100)$, and the return surface is in graph 3. Axis $\mathrm{X}$ (number of shares) means the number of stocks in the portfolio (ranged from 10-30 shares). We can see that the shape of the spectral clustering strategy is generally alike in each set of configuration. Furthermore, in our panel sample, as the number of stocks in portfolio increase, return increase significantly. 


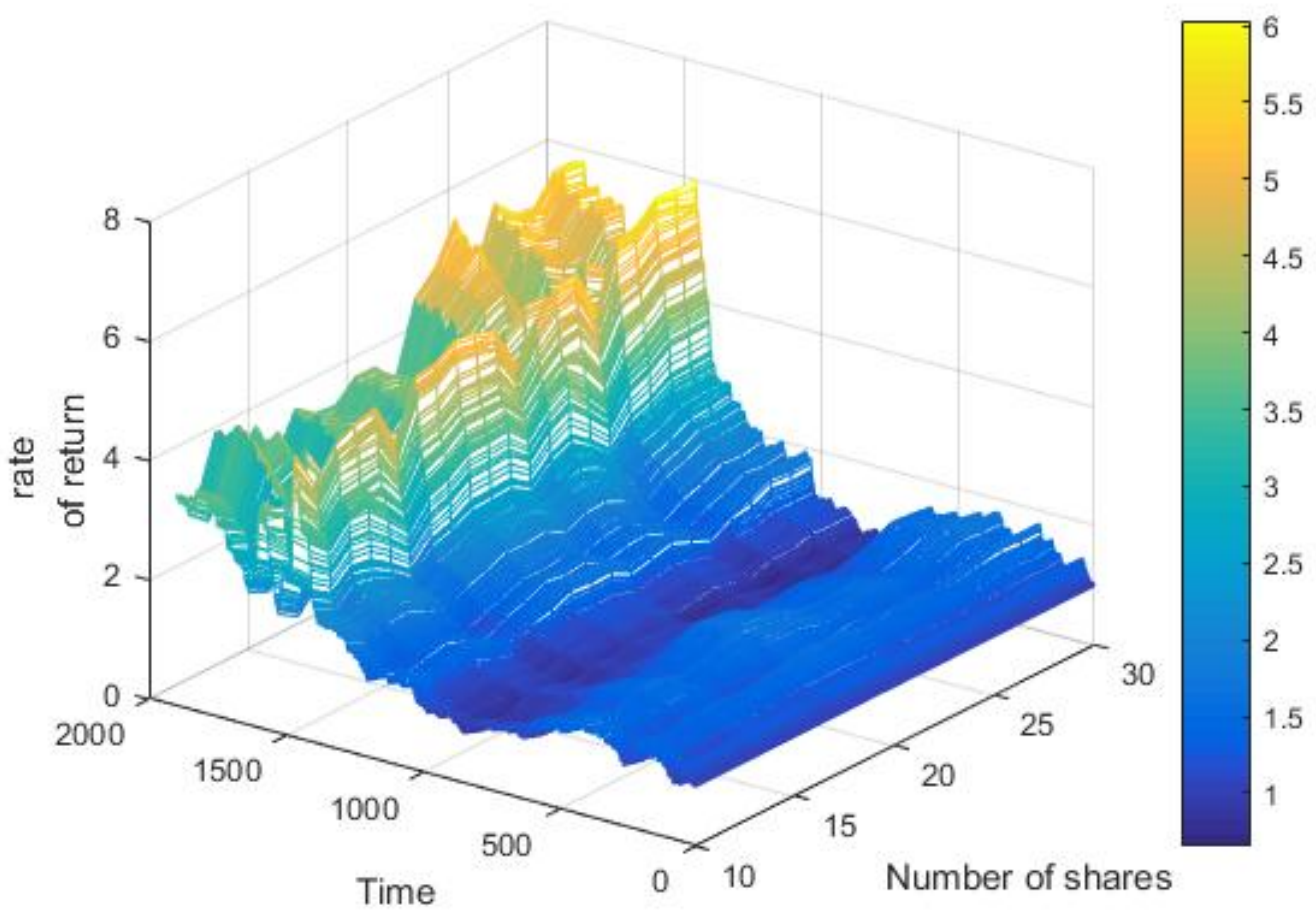

Figure 3 Return surface with $\mathrm{T}=100$

In our second robustness test, we fix the number of stocks in portfolio at 20 instead. Axis $\mathrm{X}$ is the time window used in constructing stock network, ranged from 80 days to 120 days. The result proves robustness as well: the strategy curve is generally alike under different time window. The graphs both show robustness of the strategy under different variable environment. 


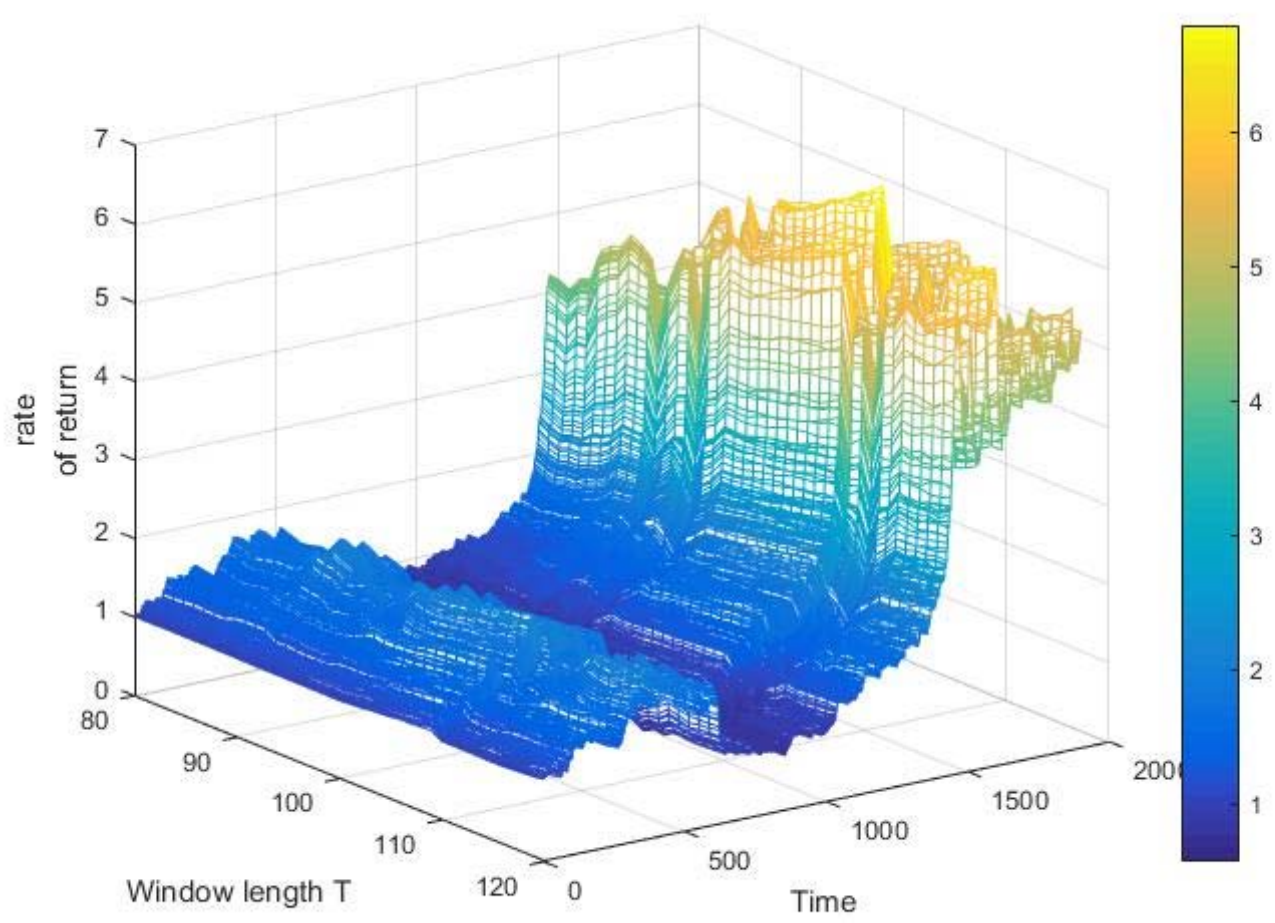

Figure 4 Return surface with $\mathrm{N}=20$

We construct an evaluation index, Dominant ratio, for better comparing spectral clustering strategy and other traditional marginalized-stock portfolio strategy, as shown as below:

$$
\text { Dominant ratio }=\sum_{R_{i j}^{S C}-R_{i j}^{T}}^{m} \operatorname{sgn}\left(R_{i j}^{S C}-R_{i j}^{T}\right) / \mathrm{M}
$$

The index gives us the ratio of time when spectral clustering strategy outperform traditional way during the holding period. $R_{i j}^{S C}$ is the return of the portfolio with $i$ stocks constructed with spectral clustering strategy in time $j$ ( the $j^{\text {th }} \Delta T$ holding time) and $R_{i j}^{T}$ is the return of the portfolio with $i$ stocks constructed with traditional strategy in time $j$. sgn(.)is a sign function and $\mathrm{M}$ is the number of $\Delta T$. The dominant ratio graph is shown as below: 


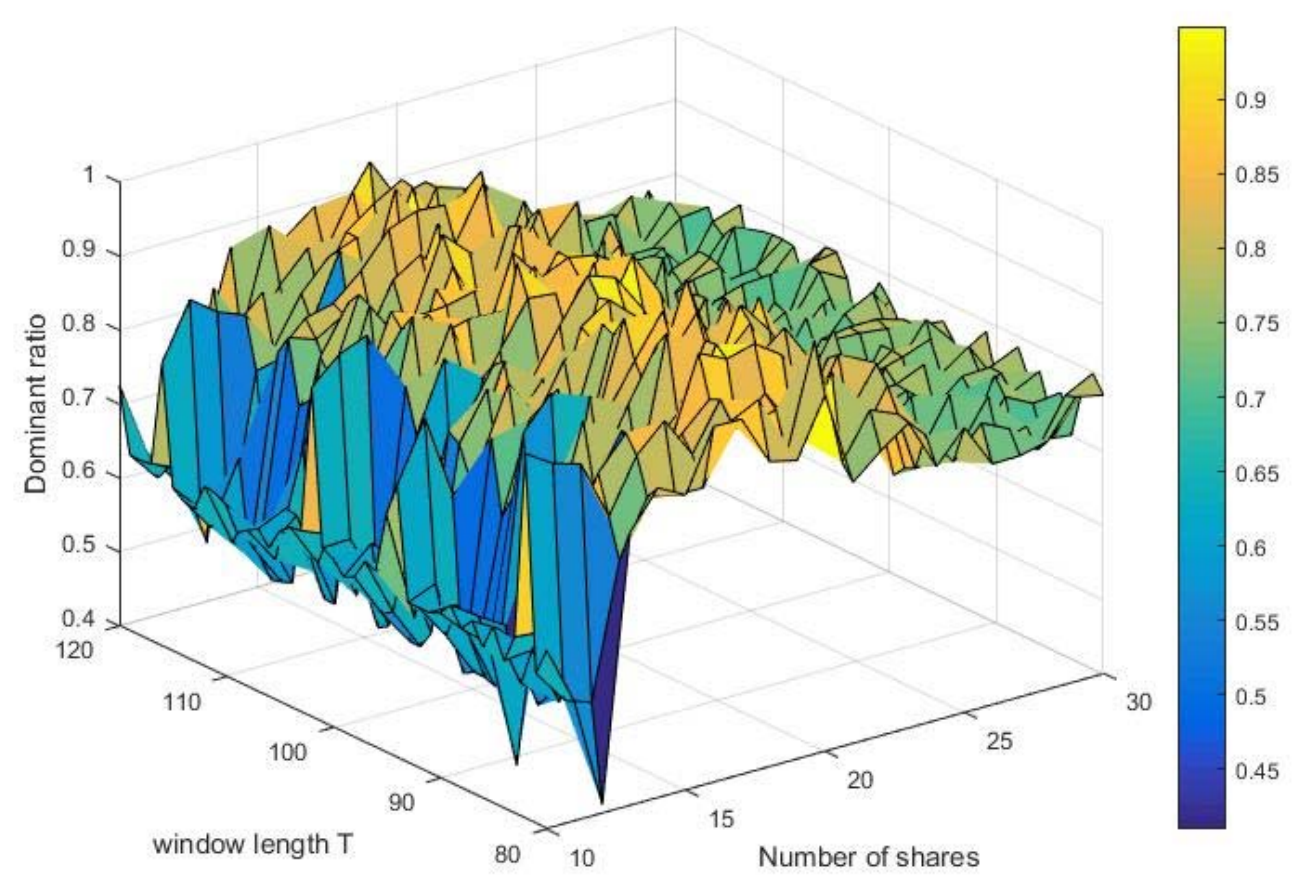

Figure 5: Dominant Ratio

Axis $\mathrm{X}$ is the amount of the stocks in portfolio, and axis $\mathrm{Y}$ is the time window of network constructing. We can find only occasional cases where dominant ratio is below 0.5 . Dominant ratio is bigger than 0.5 in most of the cases especially when number of stocks in portfolio is between 15 to 23 (steadily above 0.8 ). When the stock number is bigger than 25 , dominant ratio faces a draw back but steadily higher than 0.7 still. So in general, the spectral clustering strategy is significantly better than traditional marginalized-stock portfolio strategy. And we also prove that, this method is still robust and of good return under different occasion.

\section{Conclusion}

In this paper, we review the AI applications in different areas, especially in financial industry, point out the core techniques and specific scenes. Then we take the application in portfolio management as an example, construct a portfolio based on spectral clustering (SC) connected to stock complex network.

We construct an evolving complex network to reflect Chinese stock market by using the SC method within a moving window. We find that investments in stocks that occupy the central of the network can get higher return, especially when the network is built 
based on SC algorithm. We find that artificial intelligent algorithm can effectively enhance the investment return.

\section{References}

Akkoç S. An empirical comparison of conventional techniques, neural networks and the three stage hybrid Adaptive Neuro Fuzzy Inference System (ANFIS) model for credit scoring analysis: The case of Turkish credit card data[J]. European Journal of Operational Research, 2012, 222(1):168-178.

Bahrammirzaee A. A comparative survey of artificial intelligence applications in finance: artificial neural networks, expert system and hybrid intelligent systems[J]. Neural Computing and Applications, 2010, 19(8):1165-1195.

Bryant K. ALEES: an agricultural loan evaluation expert system[J]. Expert Systems with Applications, 2001, 21(2):75-85.

Chen L. H., Huang L., Portfolio optimization of equity mutual funds with fuzzy return rates and risks[J]. Expert Systems with Applications, 2009, 36(2):3720-3727.

Chi K. T., Liu J., Lau F. C. M, et al., A network perspective of the stock market[J]. Journal of Empirical Finance, 2010, 17(4):659-667.

Yang C. X., Shen Y. and Xia B. Y., Evaluation of Shanghai Stock Market Based On Maximal Spanning Trees[J]. Modern Physics Letters B, 2012, 27(3):50022.

Dempster M. H., Payne T. W., Romahi Y., et al. Computational learning techniques for intraday FX trading using popular technical indicators[J]. Neural Networks IEEE Transactions on, 2001, 12(4):744.

Ellis C. and Wilson P., Can a Neural Network Property Portfolio Selection Process Outperform the Property Market?[J]. Journal of Real Estate Portfolio Management, 2005(11):105-121.

Esfahanipour A, Mardani P. An ANFIS model for stock price prediction: The case of Tehran stock exchange[C], International Symposium on Innovations in Intelligent Systems and Applications. IEEE, 2011:44-49.

F. Pozzi, T. Di Matteo, and T. Aste, Spread of risk across financial markets: better to invest in the peripheries. Scientific reports, 3, 2013.

Fiedor P. Networks in financial markets based on the mutual information rate.[J]. Physical Review E Statistical Nonlinear \& Soft Matter Physics, 2014, 89(5):052801- 
052801.

Freitas F. D., Souza A. F. D. and Almeida A. R. D., Prediction-based portfolio optimization model using neural networks[J]. Neurocomputing, 2009, 72(10-12):21552170 .

Griffiths B., Beynon M. J., Expositing stages of VPRS analysis in an expert system: Application with bank credit ratings[J]. Expert Systems with Applications, 2005, 29(4):879-888.

Hamet P. and Tremblay J., Artificial Intelligence in Medicine[J]. Metabolism, 2017.

Huang Z., Chen H., Hsu C. J., et al. Credit Rating Analysis with Support Vector Machines and Neural Network: A Market Comparative Study[J]. Decision Support Systems, 2004, 37(4):543-558.

Jui-Sheng Chou, Ngoc-Tri Ngo, Wai K. Chong. Engineering Applications of Artificial Intelligence[J]. September 2016

Klein M. Finsim expert; A KB/DSS for financial analysis and planning is [J]. Engineering Costs \& Production Economics, 1989, 17(1-4):359-367.

Kwon Y. K. and Moon B. R., A Hybrid Neurogenetic Approach for Stock Forecasting[J]. IEEE Transactions on Neural Networks, 2007, 18(3):851-64.

Kodogiannis V., Lolis A. Forecasting Financial Time Series using Neural Network and Fuzzy System-based Techniques[J]. Neural Computing and Applications, 2002, 11(2):90-102.

Li X., Zhang Y., Wong H. S., et al. A hybrid intelligent algorithm for portfolio selection problem with fuzzy returns[J]. Journal of Computational \& Applied Mathematics, 2009, 233(2):264-278.

Lu C. J., Wu J. Y., Fan C. R., et al. Forecasting stock price using Nonlinear independent component analysis and support vector regression[C], IEEE International Conference on Industrial Engineering and Engineering Management. IEEE Xplore, 2009:23702374.

M. Seyedmahmoudian, B. Horan, T. Kok Soon, R. Rahmani, A. Muang Than Oo, S. Mekhilef and A. Stojcevski, State of the art artificial intelligence-based MPPT techniques for mitigating partial shading effects on PV systems - A review, Renewable and Sustainable Energy Reviews[J], Volume 64, October 2016, Pages 435-455

Ma J., Yang J., Zhang X., et al. Analysis of Chinese stock market from a complex 
network perspective: Better to invest in the central[C]// Control Conference. IEEE, 2015:8606-8611.

Majhi R., On the development of improved adaptive models for efficient prediction of stock indices using clonal-PSO (CPSO) and PSO techniques[J]. International Journal of Business Forecasting \& Marketing Intelligence, 2008, 1(1):50-67.

Makridakis S., The Forthcoming Artificial Intelligence (AI) Revolution: Its Impact on Society and Firms[J]. Futures, 2017.

Mlynarczuk M. and Skiba M.,The application of artificial intelligence for the identification of the maceral groups and mineral components of coal[J]. Computers \& Geosciences, 2017, 103:133-141.

Motiwalla L. and Wahab M., Predictable variation and profitable trading of US equities: a trading simulation using neural networks[J]. Computers \& Operations Research, 2000, 27(11-12):1111-1129.

Nair B. B. and Mohandas V. P., Artificial intelligence applications in financial forecasting --a survey and some empirical results[J]. Intelligent Decision Technologies, 2015, 9(2):99-140.

Nair B. B., Patturajan M., Mohandas V. P., et al., Predicting the BSE Sensex: Performance comparison of adaptive linear element, feed forward and time delay neural networks[C], International Conference on Power, Signals, Controls and Computation. IEEE, 2012:1-5.

Nair B. B., Sai S. G., Naveen A. N., et al., A GA-Artificial Neural Network Hybrid System for Financial Time Series Forecasting[J]. Communications in Computer \& Information Science, 2011, 147:499-506.

Oh J., Yang J. and Lee S., Managing uncertainty to improve decision-making in NPD portfolio management with a fuzzy expert system[J]. Expert Systems with Applications An International Journal, 2012, 39(10):9868-9885.

Pan Y., Heading toward Artificial Intelligence 2.0[J]. Engineering, 2016, 2(4): 409-413.

Pozzi F., Di M. T. and Aste T.. Spread of risk across financial markets: better to invest in the peripheries.[J]. Scientific Reports, 2013, 3(3):1665.

Qian Sun, Turgay Ertekin. Journal of Petroleum Science and Engineering[J], 2016

Quek C, Yow K. C., Cheng P. Y. K., et al. Investment portfolio balancing: application of a generic self-organizing fuzzy neural network (GenSoFNN)[J]. Intelligent Systems 
in Accounting Finance \& Management, 2009, 16(1-2):147-164.

R'eka Albert and Albert-L'aszl'o Barab'asi. Statistical mechanics of complex networks. Reviews of modern physics, 74(1):47, 2002.

Tsang S. W. and Jim C. Y. ,Applying artificial intelligence modeling to optimize green roof irrigation[J]. Energy \& Buildings, 2016, 127:360-369.

Vanstone B. and Finnie G., Enhancing stockmarket trading performance with ANNs[J]. Expert Systems with Applications An International Journal, 2010, 37(9):6602-6610.

Yamamoto Y. and Zenios S. A., Predicting Prepayment Rates for Mortgages Using the Cascade-Correlation Learning Algorithm[J]. Journal of Fixed Income, 1993, 2(4):8696.

Yu L., Wang S., Lai K. K., Neural network-based mean-variance-skewness model for portfolio selection[J]. Computers \& Operations Research, 2008, 35(1):34-46.

Zahraee S. M., Assadi M. K., Saidur R. Application of Artificial Intelligence Methods for Hybrid Energy System Optimization[J]. Renewable and Sustainable Energy Reviews, 2016, 66: 617-630.

Zarandi M. H. F., Zarinbal M., Ghanbari N., et al. A new fuzzy functions model tuned by hybridizing imperialist competitive algorithm and simulated annealing. Application: Stock price prediction[J]. Information Sciences, 2013, 222(3):213-228.

Zhang D., Zhou X., Leung S. C. H., et al. Vertical bagging decision trees model for credit scoring[J]. Expert Systems with Applications An International Journal, 2010, 37(12):7838-7843. 